\title{
Pegaspargase, gemcitabine, dexamethasone, and cisplatin (P-GDP) combined chemotherapy is effective for newly diagnosed extranodal NK/T- cell lymphoma: a retrospective study
}

This article was published in the following Dove Press journal:

Cancer Management and Research

Tao Liu

Fang Zhu

Yin Xiao

Qiuhui Li

Xinxiu Liu

Kunyu Yang

Gang Wu

Liling Zhang

Cancer Center, Union Hospital, Tongji Medical College, Huazhong University of Science and Technology, Wuhan,

China
Correspondence: Liling Zhang

Cancer Center, Union Hospital, Tongji

Medical College, Huazhong University

of Science and Technology, Wu jia Dun

Road I56, 430022 Wuhan, China

Tel +862785870903

Fax +862765650733

Email lily-1228@hotmail.com
Purpose: L-asparaginase or pegaspargase-based chemotherapies have shown promising results in the treatment of extranodal NK/T-cell lymphoma. A retrospective study was conducted to determine the efficacy and safety of pegaspargase, gemcitabine, dexamethasone, and cisplatin (P-GDP) combined chemotherapy in patients with newly diagnosed extranodal NK/T-cell lymphoma.

Patients and methods: From September 2013 to November 2016, 57 patients with newly diagnosed, stages I to IV, extranodal NK/T-cell lymphoma received P-GDP chemotherapy. Clinical data from these patients were collected and analyzed to evaluate the efficacy and safety of P-GDP. Results: All patients were subjected to $1-6$ cycles of P-GDP chemotherapy, and the median number of cycles of P-GDP regimen administered was 3. The overall response rate was $89.5 \%$ $(51 / 57)$, including a complete response rate of $70.2 \%$ (40/59) and a partial response rate of $19.3 \%$ (11/57). The median follow-up time was 28 months (range 2-54 months). The 2-year overall survival and progression-free survival (PFS) rates were $82.9 \%$ and $75.9 \%$, respectively. For stage I/II patients and stage III/IV patients, 2-year PFS were $80.8 \%$ and $66.7 \%$, respectively. The most common grade $3 / 4$ adverse events were neutropenia (42.1\%), thrombocytopenia (38.6\%), and hypofibrinogenemia (26.3\%). No treatment-related deaths were observed.

Conclusion: P-GDP combination chemotherapy is highly effective and safe for newly diagnosed patients with extranodal NK/T-cell lymphoma, nasal type. Additional large sample prospective trials are required to confirm these results.

Keywords: extranodal natural killer/T-cell lymphoma, pegaspargase, gemcitabine, efficacy, safety, retrospective study

\section{Introduction}

Extranodal natural killer (NK)/T-cell lymphoma, nasal type (ENKTL), is an aggressive lymphoma that is associated with the Epstein-Barr virus (EBV) and has a strong geographic predilection for Asian and Central and South American populations. ${ }^{1,2}$ Seventy to $90 \%$ of patients with ENKTL have stage I or stage II disease in the upper aerodigestive tract. ${ }^{3}$ Clinical management of early-stage ENKTL is inconsistent; chemoradiotherapy or chemotherapy with sandwiched radiotherapy has improved the prognosis recently. ${ }^{4-6}$ In addition, because NK cells express high concentrations of the multidrug-resistant P-glycoprotein (P-gp), which can actively export doxorubicin and vincristine, CHOP and CHOP-like regimens lead to inferior treatment outcomes in newly diagnosed stage IV, relapsed, or refractory ENKTL patients, who survive for less than a year. ${ }^{1,27,8}$ Recently, L-asparaginase (L-Asp)-based regimens, including SMILE and AspaMetDex, have 
been used to improve the response rate and finally benefit the overall survival (OS) and progression-free survival (PFS).$^{9-12}$ However, the drugs can lead to severe hematologic toxicity causing related infections or even death. Additionally, the short plasma half-life and allergic reactions of L-Asp also limits its use in practice. Pegaspargase (PEG-Asp), another novel drug, has proven effective against ENKTL with less toxicity and a longer half-life than L-Asp. ${ }^{13}$

Pegaspargase combined with gemcitabine, such as with GELOX/DDGP, has been demonstrated to improve the overall response rate (ORR) and survival with tolerable toxicity. $6,14,15$ However, the efficacy and safety of regimens that combine gemcitabine with pegaspargase require further investigation in additional clinical trials, especially for early-stage patients that require radiotherapy. Our previous clinical studies demonstrated that pegaspargase, gemcitabine, dexamethasone, and cisplatin (P-GDP)-combined regimens have the potential to be an effective and safe regimen for early and advanced ENKTL. ${ }^{16}$ Therefore, we conducted a retrospective study to investigate the efficacy and safety of P-GDP with or without radiotherapy for the treatment of 57 newly diagnosed ENKTL patients to test its effectiveness and safety.

\section{Patients and methods Eligibility criteria}

Newly diagnosed ENKTL patients were enrolled between September 2013 and November 2016 and were treated with a P-GDP regimen at the Cancer Center, Union Hospital, Tongji Medical College, Huazhong University of Science and Technology, Wuhan. Their pathological data were examined to confirm the diagnosis of NK/T-cell lymphoma. Immunohistochemical analyses indicated that all neoplastic cells were typically positive for cytoplasmic CD3e, CD2, CD56, CD43, granzyme B, TIA-1, and EBER, but negative for surface CD3 and CD20. Patients presented with an Eastern Cooperative Oncology Group performance status of 0-2 and had adequate hematologic, hepatic, and renal functions. Follow-up information was obtained from the patients' medical records or by telephone. This study was approved by the Ethics Committee of Tongji Medical College, Huazhong University of Science and Technology. Written informed consent was waived as there were no conflicts of interest or damage to patients and patient data confidentiality were guaranteed according to the requirements of the Ethics Committee.

\section{Disease evaluation}

Clinical evaluations at the beginning of the study included a medical history and physical examination, complete blood cell count, serum biochemistry (including hepatic function, renal function, electrolytes, lactate dehydrogenase [LDH], serum EBV DNA levels), and a bone marrow examination. Nasopharyngolaryngoscopy, magnetic resonance imaging of the head and neck, and computed tomography scans of the chest, abdomen, and pelvis areas were performed. Positron emission tomography was recommended but was not compulsory. The clinical features that were evaluated for potential prognosis included stage, disease status, B symptoms, serum LDH, International Prognostic Index (IPI) score, blood cell counts, and EBV DNA blood levels and were recorded before administration of the P-GDP regimen. All patients were staged and scored according to the Ann Arbor staging system.

\section{Study design and treatment}

All patients $(n=57)$ with newly diagnosed ENKTL were treated with the P-GDP regimen. The P-GDP protocol was repeated every 3 weeks and was administered as follows: pegaspargase $\left(2,500 \mathrm{IU} / \mathrm{m}^{2}\right.$ intramuscularly on day 4 , maximum dose 3,750 IU), gemcitabine $\left(1,000 \mathrm{mg} / \mathrm{m}^{2}\right.$ intravenously over 30 minutes on days 1 and 8 ), dexamethasone (40 mg/day orally on days $1-4)$, and cisplatin ( $25 \mathrm{mg} / \mathrm{m}^{2}$ intravenously on days $\left.1-3\right)$. For patients who were in poor condition or who experienced severe toxicities, doses were reduced accordingly. Stage I/II patients were given radiotherapy before the P-GDP regimen, and some stage III/IV patients who achieved a complete response rate (CR) or partial response (PR) after P-GDP chemotherapy received additional primary involved-field radiotherapy when the primary tumor site was in the nasal cavity or the nasopharynx. If patients developed any grade 3 nonhematological toxicities, grade 4 neutropenia complicated with fever higher than $38.5^{\circ}$, or grade 4 thrombocytopenia complicated with hemorrhage, the doses of pegaspargase and gemcitabine were then reduced by $25 \%$ in the subsequent cycles. Granulocyte colony stimulation factor (G-CSF) was administered in cases where a grade 4 neutropenia or leukopenia or a grade 3 neutropenia or leukopenia that was complicated with fever was observed. Interleukin-11 or thrombopoietin was administered in cases with grade 3/4 thrombocytopenia.

\section{Treatment response and toxicity criteria}

Revised Cheson's standard response criteria were adopted to assess the treatment response. ${ }^{17} \mathrm{~A} \mathrm{CR}$ was defined as no evidence of disease and disease-related symptoms. A PR was defined as a $\geq 50 \%$ decrease in the sum of the product of the diameters (SPDs) of masses and no new lesions. Stable disease was defined as a patient who failed to attain a CR or PR but did not fulfill those criteria for progressive disease (PD). PD was 
defined as the appearance of new sites or a $\geq 50 \%$ increase in the SPD of previous lesions. Toxicities were graded according to the National Cancer Institute Common Toxicity Criteria, version 4.0. Physical examinations and laboratory tests (routine blood test, plasma biochemical tests, and routine urine tests) were used to evaluate adverse reactions and toxicities.

\section{Statistical analysis}

OS was defined as the period from the initiation of P-GDP administration or radiotherapy to the date of the patient's death for any cause or last follow-up. PFS was defined as the period from the initiation of P-GDP administration or radiotherapy to the date of disease progression or relapse. The primary end points were the ORRs at the end of treatment. The secondary end points were CR, 2-year OS, 2-year PFS, and toxicity. The ORR was defined as the percentage of patients who achieved CR or PR. GraphPad Prism v5.0 software and SPSS statistical software v20.0 (IBM, Armonk, NY, USA) were used for the statistical analysis. Survival analysis was performed via univariate and multivariate Cox regression hazard analysis. Survival curves were derived from Kaplan-Meier survival analysis. $P$-values $<0.05$ were considered to be statistically significant.

\section{Results \\ Patient characteristics}

Fifty-seven newly diagnosed ENKTL patients were enrolled from September 2013 to November 2016 and were treated with the P-GDP regimen. Patient characteristics are summarized in Table 1 . The ratio of men to women was 1.7:1. The median age was 42 years, and a majority of patients presented with an Eastern Cooperative Oncology Group performance status of $0-1$. Thirty-nine patients $(68.4 \%)$ had stage I or stage II disease and $18(31.6 \%)$ were stage III or stage IV. The primary involvement site in 53 patients was the upper aerodigestive tract (nasal cavity, nasopharynx), while for one patient it was in the right parotid gland region, and for three patients it was in the skin. Thirty-two patients (56.1\%) had systemic B symptoms, and 16 patients $(28.1 \%)$ had elevated levels of LDH. The distribution of IPI scores included 47 patients $(82.3 \%)$ with IPI scores of $0-2$ and $10(17.7 \%)$ with IPI scores of 3-4. Thirtythree patients $(57.9 \%)$ had increased serum EBV DNA levels. Only one patient had a bone marrow infiltration.

\section{Treatment}

The total number of cycles of P-GDP regimen received by all patients was 167 , with a median of 3 cycles per patient (range, $1-6$ cycles), and pegaspargase was administered in 157 of 167 cycles. Thirty-nine stage I and II patients received involved-field
Table I Patient demographic and clinical characteristics

\begin{tabular}{|c|c|c|}
\hline \multirow[t]{2}{*}{ Characteristics } & \multicolumn{2}{|c|}{ Patients } \\
\hline & No. & $\%$ \\
\hline \multicolumn{3}{|l|}{ Gender } \\
\hline Male & 36 & 63.1 \\
\hline Female & 21 & 36.9 \\
\hline \multicolumn{3}{|l|}{ Age, years } \\
\hline$>60$ & 8 & 14.1 \\
\hline$\leq 60$ & 49 & 85.9 \\
\hline \multicolumn{3}{|l|}{ Ann Arbor Stage } \\
\hline I and II & 39 & 68.4 \\
\hline III and IV & 18 & 31.6 \\
\hline \multicolumn{3}{|l|}{ Locations of ENKTL } \\
\hline Upper aerodigestive tract & 53 & 92.9 \\
\hline Extra-upper aerodigestive tract & 4 & 7.1 \\
\hline \multicolumn{3}{|l|}{ ECOG performance status } \\
\hline $0 / 1$ & 53 & 92.9 \\
\hline 2 & 4 & 7.1 \\
\hline \multicolumn{3}{|l|}{ IPI score } \\
\hline $0-2$ & 47 & 82.3 \\
\hline $3-5$ & 10 & 17.7 \\
\hline \multicolumn{3}{|l|}{ B symptoms } \\
\hline Absent & 25 & 43.9 \\
\hline Present & 32 & 56.1 \\
\hline \multicolumn{3}{|l|}{ Serum LDH elevated } \\
\hline Normal & 41 & 71.9 \\
\hline Increased & 16 & 28.1 \\
\hline \multicolumn{3}{|l|}{ Serum EBV DNA levels (copies/ $\mu \mathrm{L}$ ) } \\
\hline$>400$ & 33 & 57.9 \\
\hline$<400$ & 24 & 42.1 \\
\hline \multicolumn{3}{|l|}{ Hemoglobin (g/L) } \\
\hline$>110$ & 38 & 66.7 \\
\hline$<110$ & 19 & 33.3 \\
\hline \multirow{2}{*}{\multicolumn{3}{|c|}{$\begin{array}{l}\text { Positron emission tomography before } \\
\text { staging }\end{array}$}} \\
\hline & & \\
\hline Yes & 46 & 80.7 \\
\hline No & 11 & 19.3 \\
\hline Bone marrow involvement & 1 & 1.8 \\
\hline \multicolumn{3}{|l|}{ Treatment } \\
\hline P-GDP alone & 11 & 19.3 \\
\hline P-GDP and radiotherapy & 46 & 80.7 \\
\hline
\end{tabular}

Abbreviations: EBV, Epstein-Barr virus; ECOG, Eastern Cooperative Oncology Group; ENKTL, extranodal natural killer/T-cell lymphoma, nasal type; IPI, International Prognostic Index; LDH, lactate dehydrogenase; P-GDP, pegaspargase, gemcitabine, dexamethasone, and cisplatin.

radiotherapy at a median dose of 56 Gy before chemotherapy. Three to four cycles of P-GDP were given to the stage I and stage II patients after radiotherapy. Stage III/IV patients with primary tumor sites in the nasal cavity or nasopharynx received radiotherapy at the end of chemotherapy while achieving a $\mathrm{CR}$ or PR. One stage IV patient received hematopoietic stem cell transplant (HSCT) after achieving a CR after chemotherapy.

\section{Efficacy and survival}

Response assessment was analyzed in 57 patients, and the results are shown in Table 2 . The ORR for all patients was 

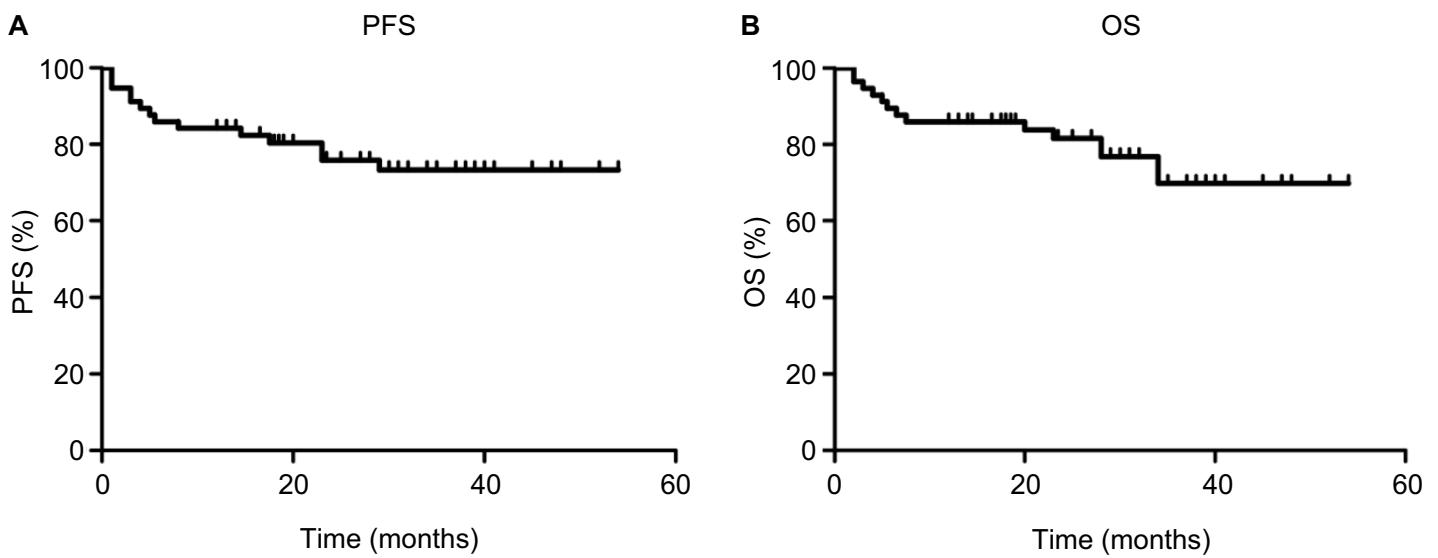

Figure I Kaplan-Meier estimates of progression-free survival (PFS) and overall survival (OS) of the entire cohort. Notes: (A) Survival analysis for PFS. The 2-year PFS rate for all patients was $75.9 \%$. (B) Survival analysis for OS. The 2 -year OS rate for all patients was $82.9 \%$.

$89.5 \%$, including $70.2 \%(40 / 57)$ in CR and $19.3 \%(11 / 57)$ in PR. The ORRs for early-stage and advanced-stage patients were $94.9 \%$ and $77.8 \%$, respectively. Stage I and II patients had an ORR of $94.9 \%$, while the ORR was $77.8 \%$ for stage III and IV patients. Furthermore, six (10.5\%) patients had the $\mathrm{PD}$, and three of them were diagnosed with hemophagocytic lymphohistiocytosis. The median follow-up time was 28 months (2-54 months) in the entire cohort. The medians for OS and PFS were not obtained. The 2-year OS and PFS rates for all patients were $82.9 \%$ and $75.9 \%$, respectively (Figure 1). Significant differences for OS and PFS were observed between stage I/II patients and stage III/IV (2-year PFS: $80.8 \%$ vs $66.7 \%, P=0.036$; 2 -year OS: $88.6 \%$ vs $66.7 \%$, $P=0.035$ ) (Table 2).

\section{Prognostic factors}

In univariate Cox regression hazard analysis (Table 3), the clinical factors that were predictive of poor survival both for PFS and OS were stage III/IV, an Eastern Cooperative Oncology Group (ECOG) score of 2, hemoglobin $<110 \mathrm{~g} / \mathrm{L}$, and an IPI score at high risk. Patients with $\leq 60$ years of age, LDH $\leq 240 \mathrm{IU} / \mathrm{L}$, or without B symptoms seemed to show better PFS and OS, but there was no significant difference. However, multivariate Cox regression hazard analysis showed that only ECOG score of 2 was predictive of a poor PFS $(\mathrm{HR}=9.857, P=0.020)$ and $\mathrm{OS}(\mathrm{HR}=8.528, P=0.035)$ (Table 4). The results are shown in Figure 2.

\section{Toxicity}

Treatment-related toxicities are summarized in Table 5. The most common grade 3-4 hematologic adverse events were neutropenia ( $\mathrm{n}=24,42.1 \%)$, leukopenia ( $\mathrm{n}=28,49.1 \%)$, and thrombocytopenia ( $\mathrm{n}=22,38.6 \%)$. Grade 3 hypofibrinogen-
Table 2 Results of ORR, PFS, and OS with P-GDP regimen in ENKTL

\begin{tabular}{|l|l|l|l|}
\hline \multirow{2}{*}{ Response } & \multicolumn{3}{|l|}{ No. of patients (\%) } \\
\cline { 2 - 4 } & $\begin{array}{l}\text { Total patient } \\
(\mathbf{n}=\mathbf{5 7})\end{array}$ & $\begin{array}{l}\text { Stage I and } \\
\text { II (n=39) }\end{array}$ & $\begin{array}{l}\text { Stage III and } \\
\text { IV (n= I 8) }\end{array}$ \\
\hline CR & $40(70.2)$ & $32(82.1)$ & $8(44.4)$ \\
PR & II (19.3) & $5(I 2.8)$ & $6(33.3)$ \\
SD & $0(0)$ & $0(0)$ & $0(0)$ \\
PD & $6(10.5)$ & $2(5.1)$ & $4(22.2)$ \\
ORR & $5 I(89.3)$ & $37(94.9)$ & $14(77.8)$ \\
2-year PFS & $43(75.9)$ & $32(80.8)$ & $12(66.7)$ \\
2-year OS & $47(82.9)$ & $35(88.6)$ & $12(66.7)$ \\
\hline
\end{tabular}

Abbreviations: CR, complete response; ENKTL, extranodal natural killer/T-cell lymphoma, nasal type; ORR, overall response rate ; OS, overall survival; P-GDP, pegaspargase, gemcitabine, dexamethasone, and cisplatin; $\mathrm{PD}$, progressive disease; PFS, progression-free survival; PR, partial remission; SD, stable disease.

emia was observed in 15 (26.3\%) patients and was the most common nonhematological grade 3-4 toxicity. Two patients and one patient had to have their chemotherapy suspended due to grade 3 hyperbilirubinemia and increased transaminases, respectively. Grade 1-2 increased transaminases and hypoalbuminemia were the most common nonhematological toxicities. Three patients were taken off treatment with pegaspargase because of thrombosis $(n=2)$ or an allergy to pegaspargase $(n=1)$, and eight patients had a dose reduction or delay because of severe hypofibrinogenemia $(n=2)$, an abnormal liver function $(n=1)$, or hyperbilirubinemia $(n=2)$ or hypoalbuminemia $(\mathrm{n}=2)$. All adverse events were manageable with supportive care. No pancreatitis was observed and no treatment-related deaths occurred.

\section{Discussion}

This is the largest number of cases combining P-GDP chemotherapy for the treatment of newly diagnosed extranodal 
Table 3 Univariate analysis of the association between clinical variables with OS and PFS for all patients with ENKTL

\begin{tabular}{|c|c|c|c|c|}
\hline \multirow[t]{2}{*}{ Factors } & \multicolumn{2}{|l|}{ PFS } & \multicolumn{2}{|l|}{ OS } \\
\hline & HR (95\% Cl) & $P$-value & HR (95\% Cl) & $P$-value \\
\hline Gender, female vs male & $1.526(0.476-4.868)$ & 0.475 & I.56I (0.489-4.987) & 0.452 \\
\hline Age $>60$ vs $\leq 60$ years & $1.98 I(0.55 I-7.128)$ & 0.295 & $2.147(0.596-7.735)$ & 0.243 \\
\hline Stage III/IV vs I/II & 2.918 (1.017-8.369) & $0.046^{*}$ & $3.025(|.05|-8.7 \mid 2)$ & $0.040^{*}$ \\
\hline ECOG PS 2 vs $0 / 1$ & $21.034(5.022-88.100)$ & $0.000^{*}$ & $20.236(4.904-83.507)$ & $0.000^{*}$ \\
\hline B symptoms, yes vs no & $3.086(0.860-11.073)$ & 0.084 & $3.032(0.845-10.880)$ & 0.089 \\
\hline $\mathrm{LDH},>240$ vs $\leq 240 \mathrm{IU} / \mathrm{L}$ & $2.55 I(0.88 I-7.39 I)$ & 0.084 & $2.83 \mathrm{I}(0.972-8.245)$ & 0.056 \\
\hline IPI score, $3-5$ vs $0-2$ & $4.332(1.423-13.187)$ & $0.010^{*}$ & $4.68(1.528-14.338)$ & $0.007^{*}$ \\
\hline $\begin{array}{l}\text { EBV DNA levels, } \geq 400 \\
\text { vs }<400 \text { copies } / \mu \mathrm{L}\end{array}$ & I. $145(0.396-3.316)$ & 0.802 & $1.159(0.400-3.353)$ & 0.786 \\
\hline $\begin{array}{l}\text { Hemoglobin, } \geq 110 \text { vs } \\
<110 \text { g/L }\end{array}$ & $3.090(|.07|-8.9 \mid 8)$ & $0.037^{*}$ & $3.129(1.084-9.030)$ & $0.035^{*}$ \\
\hline
\end{tabular}

Note: $* P<0.05$, the difference was statistically significant.

Abbreviations: EBV, Epstein-Barr virus; ECOG, Eastern Cooperative Oncology Group; ENKTL, extranodal natural killer/T-cell lymphoma, nasal type; IPI, International Prognostic Index; LDH, lactate dehydrogenase; OS, overall survival; PFS, progression-free survival; PS, performance status.

Table 4 Multivariate analysis of the association between clinical variables with OS and PFS for all patients with ENKTL

\begin{tabular}{|l|l|l|l|l|}
\hline \multirow{2}{*}{ Factors } & \multicolumn{2}{|l|}{ PFS } & OS \\
\cline { 2 - 5 } & HR (95\% Cl) & P-value & HR (95\% Cl) & P-value \\
\hline Stage III/IV vs I/II & $1.106(0.214-5.708)$ & 0.904 & $1.159(0.228-5.89 I)$ & 0.859 \\
ECOG PS 2 vs 0/I & $9.857(1.437-67.622)$ & $0.020^{*}$ & $8.528(1.159-62.733)$ & $0.035 *$ \\
IPI score 3-5 vs 0-2 & $1.646(0.277-9.799)$ & 0.584 & $1.706(0.258-11.272)$ & 0.579 \\
Hemoglobin & $1.670(0.470-5.935)$ & 0.428 & $1.744(0.488-6.233)$ & 0.392 \\
$\geq 110$ vs $<110$ g/L & & & & \\
\hline
\end{tabular}

Note: $* P<0.05$, the difference was statistically significant.

Abbreviations: ECOG, Eastern Cooperative Oncology Group; ENKTL, extranodal natural killer/T-cell lymphoma, nasal type; IPI, International Prognostic Index; OS, overall survival; PFS, progression-free survival; PS, performance status.

NK/T-cell lymphoma. ${ }^{14,15}$ This retrospective study enrolled 57 newly diagnosed extranodal NK/T-cell lymphoma patients to test the effectiveness and toxicity of a P-GDP regimen with or without radiotherapy. In particular, this was the first study to treat early-stage ENKTL using a P-GDP regimen combined with radiotherapy. The ORR was $89.3 \%$ with a $70.2 \% \mathrm{CR}$ rate. The 2-year PFS and 2-year OS rates were $75.9 \%$ and $82.9 \%$. The most common grade $3-4$ adverse events included neutropenia (42.1\%), thrombocytopenia (38.6\%), and hypofibrinogenemia $(26.3 \%)$. These results are similar or superior to the response rates, survivals, and toxicities that have been reported in previous studies of chemotherapy followed, or not, by radiotherapy. ${ }^{6,11,14,15,18}$ This study demonstrates that the P-GDP regimen is an effective and tolerable treatment strategy for this newly diagnosed patient population combined with or without radiotherapy.

For stages IE to IIE nasal ENKTL patients, concurrent chemoradiation therapy, sequential chemoradiation, or sandwich chemoradiation are the common strategies for the treatment, while chemotherapy is a mainstay treatment for patients with advanced-stage, relapsed, or refractory ENKTL. However, the optimal chemotherapy regimen is still controversial. Recently, a number of L-Asp-based regimens, such as SMILE and AspaMetDex, have yielded promising results in patients with advanced-stage or relapsed/ refractory ENKTL. ${ }^{10,11,18}$ Moreover, several case reports and clinical studies suggest that pegaspargase is a potentially effective agent when used in the treatment of ENKTL. ${ }^{6,15,18}$ Pegaspargase, in particular, which is associated with a lower incidence of antiasparaginase antibody induction and exhibits more prolonged asparaginase activity than native L-Asp, is an optimal drug for ENKTL. ${ }^{8}$ Gemcitabine, an analog of cytosine arabinoside, is widely used to treat solid tumors and is effective against lymphoma, particularly in relapsed/ refractory patients. ${ }^{19,20}$ Park et al reported that GDP was a highly effective and optional salvage regimen for relapsed/ refractory peripheral $\mathrm{T}$ cell lymphomas (PTCLs). The ORR was $72 \%$ with $48 \%$ CR rate in a prospective phase II study. ${ }^{21}$ For the treatment of ENKTL, Ahn et al reported that using gemcitabine alone or with chemotherapy for relapsed/refrac- 

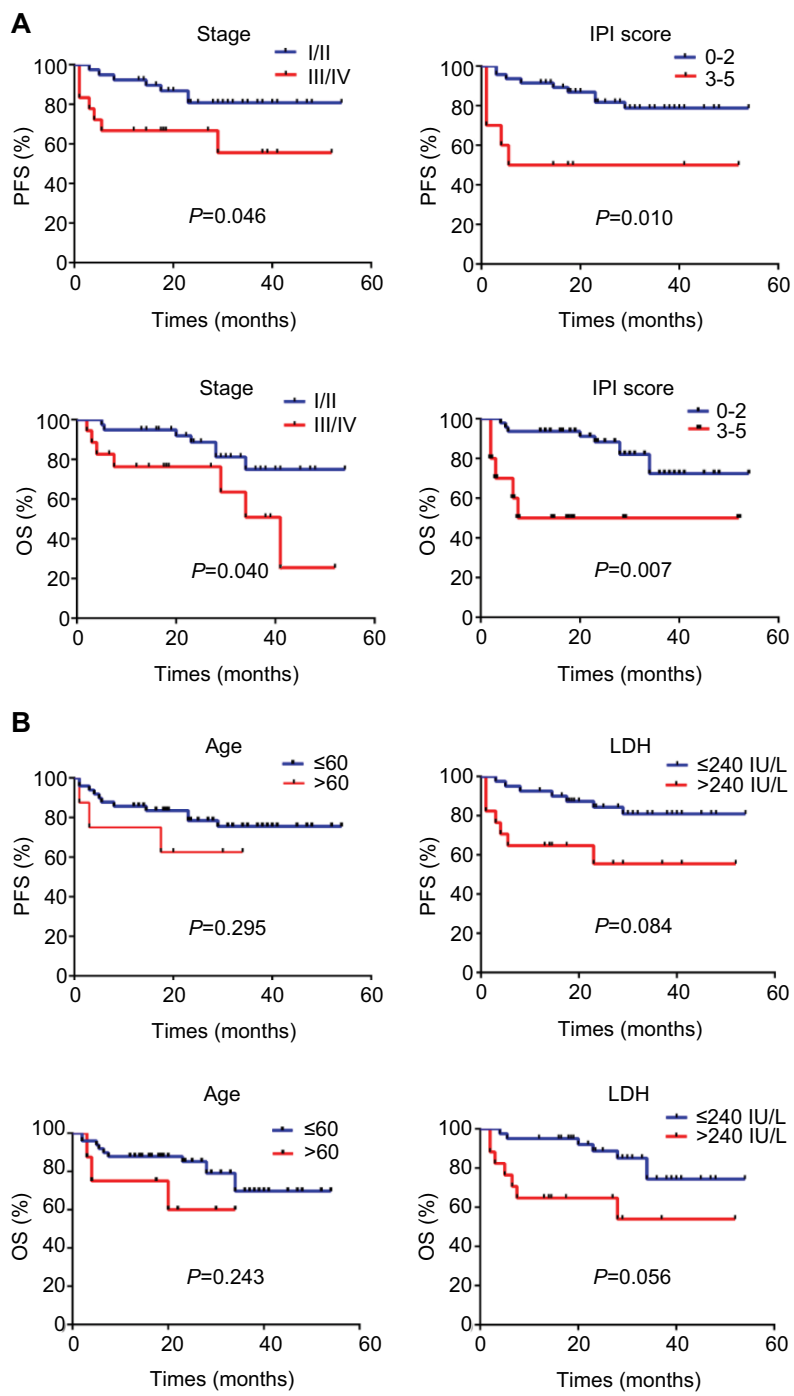
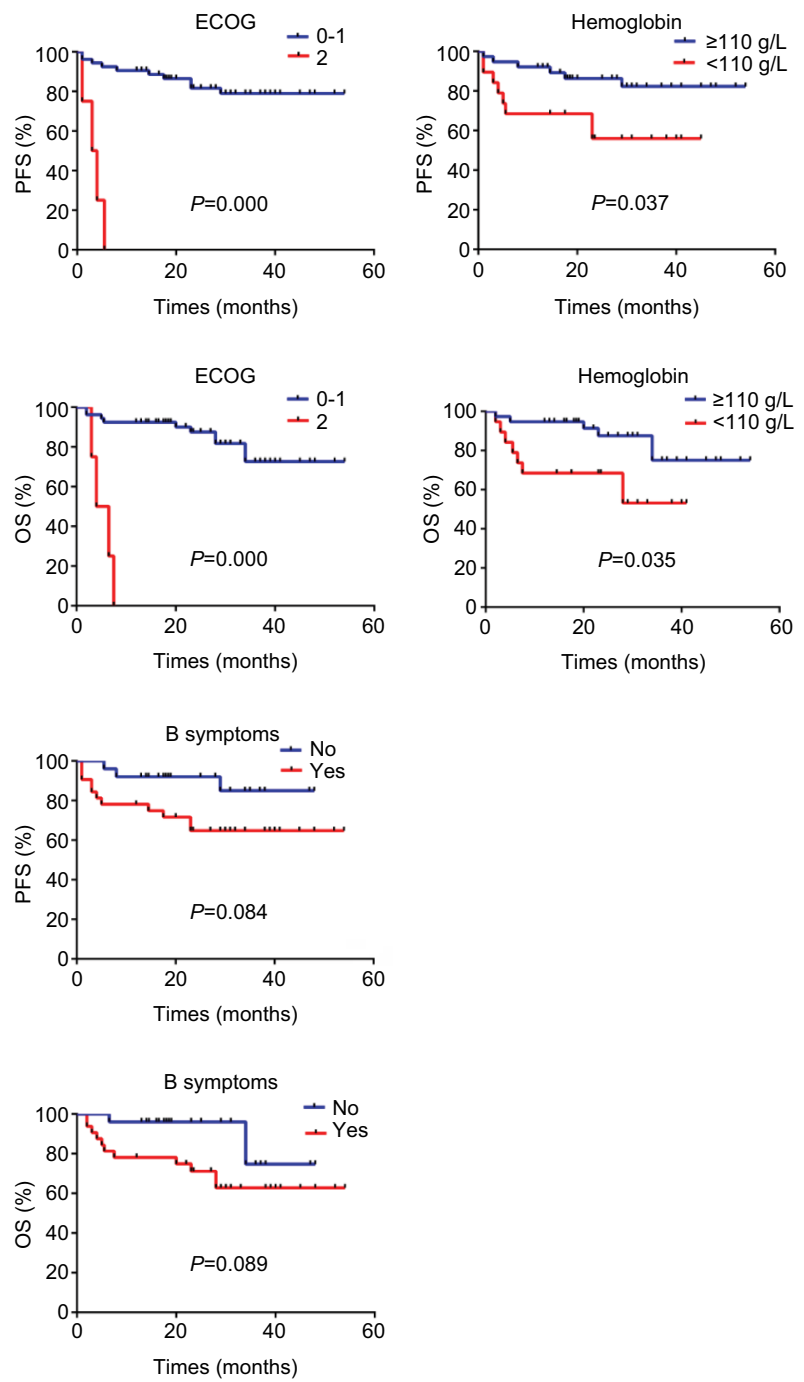

Figure 2 Comparison of PFS and OS according to prognostic factors.

Notes: (A) Patients with stage III/IV disease, an ECOG score of 2, hemoglobin $<110 \mathrm{~g} / \mathrm{L}$, or an IPI score at high risk showed poor survival for PFS and OS. (B) Patients $\leq 60$ years of age, $\mathrm{LDH} \leq 240 \mathrm{IU} / \mathrm{L}$, or without $\mathrm{B}$ symptoms showed better PFS and OS, but there was no significant difference.

Abbreviations: ECOG, Eastern Cooperative Oncology Group; IPI, International Prognostic Index; LDH, lactate dehydrogenase; OS, overall survival; PFS, progression-free survival.

tory disease, eight of 20 patients $(40 \%)$ achieved a response. ${ }^{19}$ In addition, Wang et al reported a GDP regimen that yielded an ORR of $83 \%$ in newly diagnosed stage IV and relapsed/ refractory patients. ${ }^{14}$ In a previous phase II clinical trial to evaluate the efficacy and safety of GELOX as an induction therapy for early-stage ENKTL, a 3-year OS rate of 78.0\% with acceptable safety profiles was observed. ${ }^{6}$ These studies suggest that pegaspargase and gemcitabine combined regimens maybe an effective and safe strategy.

Patients with advanced-stage and relapsed/refractory disease have a poor prognosis, and no standard management has been identified. Table 6 summarizes the latest published results using L-Asp/pegaspargase or gemcitabine-based regimens for this disease. As shown in the table, in newly diagnosed stage III/IV patients, ORR (77.8\%) and CR rates $(44.4 \%)$ were obtained. It is evident that the treatment responses and survival outcomes that were obtained with the P-GDP regimen are similar to those achieved with the SMILE and AspaMetDex regimens and that patient survival was improved, ${ }^{10,11,18}$ which is indicative of the excellent antitumor effect of the P-GDP regimen. However, the response rate and survival results from our study are inferior to those reported by previously published studies. ${ }^{15,22}$ Possible reasons for this difference are that the sample size of advanced-stage patients was too small (only 18) in our study, and moreover, that four $(22.2 \%)$ patients presented in poor physical condition, which is associated with poor response rates and short survivals. Nevertheless, our results suggest that the P-GDP regimen 
can be a potential option for newly diagnosed advanced-stage ENKTL patients.

For stage I/II ENKTL patients, treatments include radiotherapy alone or with combined modalities. A phase II trial of concurrent chemotherapy and radiotherapy (CCRT) followed by three cycles of VIPD was conducted to determine the efficiency of chemotherapy after radiotherapy in newly diagnosed, stage IE to IIE, ENKTL patients. ${ }^{4}$ The estimated

Table 5 P-GDP-related toxicity profiles

\begin{tabular}{|l|l|l|}
\hline Toxicity & \multicolumn{2}{|l|}{$\begin{array}{l}\text { Toxicity incidence, } \\
\text { No. (\%) }\end{array}$} \\
\cline { 2 - 3 } & $\begin{array}{l}\text { All grade } \\
\text { I-4 }\end{array}$ & $\begin{array}{l}\text { Grade } \\
\text { 3/4 }\end{array}$ \\
\hline Hematologic & & \\
Leukopenia & $55(96.5)$ & $28(49.1)$ \\
Neutropenia & $46(8 I .7)$ & $24(42.1)$ \\
Anemia & $54(94.7)$ & $0(0)$ \\
Thrombocytopenia & $42(73.7)$ & $22(38.6)$ \\
Nonhematologic & & \\
Hypofibrinogenemia & $42(73.7)$ & $15(26.3)$ \\
APTT elongation & $25(67.6)$ & $0(0)$ \\
Hyperbilirubinemia & $29(78.4)$ & $2(3.5)$ \\
Increased transaminases & $3 I(83.8)$ & $1(1.8)$ \\
Hypoalbuminemia & $37(100)$ & $0(0)$ \\
Hypertriglyceridemia & $19(5 I .4)$ & $0(0)$ \\
Increased BUN & $4(10.8)$ & $0(0)$ \\
Thrombosis & $2(5.4)$ & $0(0)$ \\
Allergy & $I(2.7)$ & $0(0)$ \\
Gastrointestinal disorders & $I 2(32.4)$ & $0(0)$ \\
Increased serum amylase & $I(2.7)$ & $0(0)$ \\
Hyperglycemia & $30(52.6)$ & $0(0)$ \\
\hline
\end{tabular}

Abbreviations: APTT, activated partial thromboplastin time; BUN, blood urea nitrogen; P-GDP, pegaspargase, gemcitabine, dexamethasone, and cisplatin. 3-year PFS and OS were 85.2\% (95\% CI, 72.5\%-97.9\%) and $86.3 \%$ (95\% CI, 74.0\%-98.6\%), respectively. A 100\% response rate was attained that included $22(73.3 \%) \mathrm{CR}$ and 8 (26.7\%) PR. In another trial, 19 patients received sandwiched radiotherapy at a median dose of 50 (30-52) Gy with SMILE regimens. The ORR was 79\%, the 1 -year PFS was 53\%, and the 1 -year OS was $55 \%$, while $67 \%$ of patients had grade $3 / 4$ neutropenia. ${ }^{11} \mathrm{Bi}$ et al reported nine patients with stage I-II disease who were treated with PGEMOX or GELOX followed by RT that had an encouraging outcome (5-year OS 100\%, 5-year PFS $85.7 \%$ ), which was superior to that of patients receiving other regimens followed by $\mathrm{RT}^{6}{ }^{6}$ However, the number of patients enrolled was small. The results of our study for 39 newly diagnosed stage I/II ENKTL patients after CCRT followed by P-GDP are shown in Table 6; the ORR was $94.9 \%$ and the CR was $82.1 \%$. The survival was also superior to SMILE combined with radiotherapy, while it was similar to PGEMOX/GELOX or VIPD therapies after CCRT. Furthermore, the grade 3/4 neutropenia was relatively lower compared with SMILE and VIPD regimens. Thus, CCRT followed by $\mathrm{P}-\mathrm{GDP}$ produced promising response rates and survival for newly diagnosed stage I/II ENKTL patients.

In this study, grade 1-2 toxicities had frequent side effects, but they could be well controlled with supportive treatments. Grade 3/4 toxicities were mainly hematological. Timely administration of G-CSF was essential when patients experienced severe cytopenia after chemotherapy. Grade 3 hypofibrinogenemia was another adverse event without severe coagulopathies and grade 4 hypofibrinogenemia. Other commonly reported side effects of pegaspargase therapy include

Table 6 Studies of pegaspargase- or L-asparaginase-based regimens and gemcitabine-based regimens in the treatment of ENKTL

\begin{tabular}{|c|c|c|c|c|c|c|c|c|c|}
\hline \multirow[t]{2}{*}{ Disease status } & \multirow{2}{*}{$\begin{array}{l}\text { Treatment } \\
\text { and } \\
\text { regimens }\end{array}$} & \multirow[t]{2}{*}{ No. } & \multirow{2}{*}{$\begin{array}{l}\text { Stage } \\
\text { I-II }\end{array}$} & \multirow[b]{2}{*}{ III-IV } & \multicolumn{2}{|c|}{ Response } & \multicolumn{2}{|l|}{ Survival } & \multirow{2}{*}{$\begin{array}{l}\begin{array}{l}\text { Adverse } \\
\text { effects }\end{array} \\
\text { Grade } 3 / 4 \\
\text { neutropenia }\end{array}$} \\
\hline & & & & & $\begin{array}{l}\text { ORR } \\
\text { (\%) }\end{array}$ & $\begin{array}{l}\text { CR } \\
\text { (\%) }\end{array}$ & PFS & os & \\
\hline $\mathrm{R} / \mathrm{R}^{9}$ & AspaMetDex & 19 & $12(63 \%)$ & 7 (27\%) & 78 & 61 & 2 years: $40 \%$ & $\begin{array}{l}2 \text { years: } \\
40 \%\end{array}$ & $42 \%$ \\
\hline $\begin{array}{l}\text { Newly diagnosed } \\
\text { stage } I V \text { and } R / R^{10}\end{array}$ & SMILE & 38 & II (29\%) & 27 (7।\%) & 79 & 45 & I year :53\% & $\begin{array}{l}\text { I year: } \\
55 \%\end{array}$ & $100 \%$ \\
\hline $\begin{array}{l}\text { Newly diagnosed } \\
\text { advanced stage }^{21}\end{array}$ & DDGP & 21 & & $21(100 \%)$ & 95 & 71 & I year: $86 \%$ & $\begin{array}{l}2 \text { years: } \\
74 \%\end{array}$ & $15 \%$ \\
\hline $\begin{array}{l}\text { Newly diagnosed } \\
\text { stage IV and } R / R^{13}\end{array}$ & GDP & 41 & 7 (I7.1\%) & 34 (82.9\%) & 83 & 41.5 & $\begin{array}{l}\text { I year: } \\
54,5 \%\end{array}$ & $\begin{array}{l}\text { I year: } \\
72.7 \%\end{array}$ & $34.1 \%$ \\
\hline $\begin{array}{l}\text { Newly diagnosed and } \\
R / R^{5}\end{array}$ & $\begin{array}{l}\text { SMILE } \pm \\
\text { sandwiched } \\
\text { RT (50 Gy) }\end{array}$ & 87 & $38(43 \%)$ & 49 (56\%) & 81 & 66 & $\begin{array}{l}4 \text { years DFS: } \\
64 \%\end{array}$ & $\begin{array}{l}5 \text { years: } \\
50 \%\end{array}$ & $67 \%$ \\
\hline $\begin{array}{l}\text { Newly diagnosed } \\
\text { (present study) }\end{array}$ & $\begin{array}{l}P-G D P \pm R T \\
(56 \mathrm{~Gy})\end{array}$ & 57 & 39 (68.4\%) & $18(31.6 \%)$ & 89.3 & 70.2 & $\begin{array}{l}2 \text { years: } \\
75.9 \%\end{array}$ & $\begin{array}{l}2 \text { years: } \\
82.9 \%\end{array}$ & $42.1 \%$ \\
\hline
\end{tabular}

Abbreviations: AspaMetDex, L-asparaginase, methotrexate, dexamethasone; CR, complete response; DDGP, gemcitabine, pegaspargase, cisplatin, and dexamethasone; DFS, disease-free survival; ENKTL, extranodal natural killer/T-cell lymphoma, nasal type; GDP, gemcitabine, dexamethasone, and cisplatin; ORR, overall response rates; OS, overall survival; PFS, progression-free survival; R/R, relapsed/refractory; RT, radiotherapy; SMILE, steroid, methotrexate, ifosfamide, L-asparaginase, and etoposide. 
liver dysfunction, hypoalbuminemia, hypertriglyceridemia, gastrointestinal disorders, and hyperglycemia. Venous thrombosis and allergies are other relatively common adverse events of asparaginase; two patients developed venous thrombosis during our study period, and one patient was allergic to asparaginase after its use. There was no pancreatitis observed in this study, and no treatment-related deaths occurred. The hematologic toxicities and liver dysfunctions that were observed in our study were considerably milder than those reported with the SMILE and AspaMetDex regimens. ${ }^{10,11,18}$ In summary, the P-GDP regimen presented acceptable toxicity profiles.

In conclusion, this study demonstrated that P-GDP is an effective and well-tolerated treatment for patients with newly diagnosed ENKTL with or without radiotherapy. However, this is a retrospective study with a relatively small sample size. Prospective randomized clinical trials will be required to validate the long-term efficacy of the P-GDP regimen.

\section{Conclusion}

This study contains the largest number of cases that combine P-GDP for chemotherapy in the treatment of newly diagnosed ENKTL. P-GDP is an effective and well-tolerated treatment for patients with newly diagnosed ENKTL compared with current treatment options. Additional large sample prospective trials are required to confirm the long-term efficacy of P-GDP with or without radiotherapy in newly diagnosed ENKTL patients.

\section{Acknowledgment}

We acknowledge the support received from the National Natural Science Foundation of China (81672940, 81602696)

\section{Author contributions}

LZ and GW conceived and designed the project. FZ, YX, $\mathrm{XL}$, and TL performed patient collection and clinical data interpretation. TL and QL performed the statistical analyses. TL, KY, and LZ wrote the manuscript. All authors contributed toward data analysis, drafting and critically revising the paper and agree to be accountable for all aspects of the work.

\section{Disclosure}

The authors report no conflicts of interest in this work.

\section{References}

1. Tse E, Kwong YL. How I treat NK/T-cell lymphomas. Blood. 2013;121(25):4997-5005.

2. Au WY, Weisenburger DD, Intragumtornchai $\mathrm{T}$, et al. Clinical differences between nasal and extranasal natural killer/T-cell lymphoma: a study of 136 cases from the International Peripheral T-Cell Lymphoma Project. Blood. 2009;113(17):3931-3937.
3. Au WY, Ma SY, Chim CS, et al. Clinicopathologic features and treatment outcome of mature T-cell and natural killer-cell lymphomas diagnosed according to the World Health Organization classification scheme: a single center experience of 10 years. Ann Oncol. 2005;16(2):206-214.

4. Kim SJ, Kim K, Kim BS, et al. Phase II trial of concurrent radiation and weekly cisplatin followed by VIPD chemotherapy in newly diagnosed, stage IE to IIE, nasal, extranodal NK/T-cell lymphoma: Consortium for Improving Survival of Lymphoma study. J Clin Oncol. 2009;27(35):6027-6032.

5. Yamaguchi M, Tobinai K, Oguchi M, et al. Concurrent chemoradiotherapy for localized nasal natural killer/T-cell lymphoma: an updated analysis of the Japan clinical oncology group study JCOG0211. J Clin Oncol. 2012;30(32):4044-4046.

6. Bi XW, Xia Y, Zhang WW, et al. Radiotherapy and PGEMOX/GELOX regimen improved prognosis in elderly patients with early-stage extranodal NK/T-cell lymphoma. Ann Hematol. 2015;94(9):1525-1533.

7. Kwong YL. Natural killer-cell malignancies: diagnosis and treatment. Leukemia. 2005;19(12):2186-2194.

8. Yamaguchi M, Kita K, Miwa H, et al. Frequent expression of P-glycoprotein/MDR1 by nasal T-cell lymphoma cells. Cancer. 1995;76(11):2351-2356.

9. Jaccard A, Gachard N, Marin B, et al. Efficacy of L-asparaginase with methotrexate and dexamethasone (AspaMetDex regimen) in patients with refractory or relapsing extranodal NK/T-cell lymphoma, a phase 2 study. Blood. 2011;117(6):1834-1839.

10. Yamaguchi M, Kwong YL, Kim WS, et al. Phase II study of SMILE chemotherapy for newly diagnosed stage IV, relapsed, or refractory extranodal natural killer (NK)/T-cell lymphoma, nasal type: the NK-Cell Tumor Study Group study. J Clin Oncol. 2011;29(33):4410-4416.

11. Kwong YL, Kim WS, Lim ST, et al. SMILE for natural killer/T-cell lymphoma: analysis of safety and efficacy from the Asia Lymphoma Study Group. Blood. 2012;120(15):2973-2980.

12. Yamaguchi M, Suzuki R, Oguchi M. Advances in the treatment of extranodal NK/T-cell lymphoma, nasal type. Blood. 2018;131(23):2528-2540.

13. Escherich G, Zur Stadt U, Zimmermann M, Horstmann MA, CoALL Study Group. Clofarabine in combination with pegylated asparaginase in the frontline treatment of childhood acute lymphoblastic leukaemia: a feasibility report from the CoALL 08-09 trial. Br J Haematol. 2013;163(2):240-247.

14. Wang JJ, Dong M, He XH, et al. GDP (gemcitabine, dexamethasone, and cisplatin) is highly effective and well-tolerated for newly diagnosed stage IV and relapsed/refractory extranodal natural killer/T-cell lymphoma, nasal type. Medicine. 2016;95(6):e2787.

15. Zhou Z, Li X, Chen C, et al. Effectiveness of gemcitabine, pegaspargase, cisplatin, and dexamethasone (DDGP) combination chemotherapy in the treatment of relapsed/refractory extranodal NK/T cell lymphoma: a retrospective study of 17 patients. Ann Hematol. 2014;93(11):1889-1894.

16. Zhang L, Zhu F, Xiao Y, et al. Phase II study of concurrent chemoradiotherapy followed by Peg-Asp-GDP for localized nasal NK/T-cell lymphoma: a prospective single-center clinical trial. Hematol Oncol. 2015;33:299.

17. Cheson BD, Pfistner B, Juweid ME, et al. Revised response criteria for malignant lymphoma. J Clin Oncol. 2007;25(5):579-586.

18. Yamaguchi M, Suzuki R, Kwong YL, et al. Phase I study of dexamethasone, methotrexate, ifosfamide, L-asparaginase, and etoposide (SMILE) chemotherapy for advanced-stage, relapsed or refractory extranodal natural killer (NK)/T-cell lymphoma and leukemia. Cancer Sci. 2008;99(5):1016-1020.

19. Ahn HK, Kim SJ, Hwang DW, et al. Gemcitabine alone and/or containing chemotherapy is efficient in refractory or relapsed NK/T-cell lymphoma. Invest New Drugs. 2013;31(2):469-472.

20. Sallah S, Wan JY, Nguyen NP. Treatment of refractory T-cell malignancies using gemcitabine. Br J Haematol. 2001;113(1):185-187.

21. Park BB, Kim WS, Suh C, et al. Salvage chemotherapy of gemcitabine, dexamethasone, and cisplatin (GDP) for patients with relapsed or refractory peripheral T-cell lymphomas: a consortium for improving survival of lymphoma (CISL) trial. Ann Hematol. 2015;94(11):1845-1851.

22. Li X, CuiY, Sun Z, et al. DDGP versus SMILE in newly diagnosed advanced natural killer/T-cell lymphoma: a randomized controlled, multicenter, openlabel study in China. Clin Cancer Res. 2016;22(21):5223-5228. 
Cancer Management and Research

\section{Publish your work in this journal}

Cancer Management and Research is an international, peer-reviewed open access journal focusing on cancer research and the optimal use of preventative and integrated treatment interventions to achieve improved outcomes, enhanced survival and quality of life for the cancer patient.

The manuscript management system is completely online and includes

Submit your manuscript here: https://www.dovepress.com/cancer-management-and-research-journal 